\title{
Automatic Semantic Sequence Extraction from Unrestricted Non-Tagged Texts
}

\author{
Shiho Nobesawa and Hiroaki Saito and Masakazu Nakanishi \\ Dept. of Computer Science \\ Keio University \\ 3-14-1 Hiyoshi Kohoku, Yokohama 223-8522, Japan \\ \{shiho, hxs, czl\}@nak.ics.keio.ac.jp
}

\begin{abstract}
Mophological processing, syntactic parsing and other uscful tools have been proposed in the field of natural language processing(NLP). Many of those NLP tools take dictionary-based approaches. Thus these tools are often not very efficicnt with texts written in casual wordings or texts which contain many domain-specific terms, because of the lack of vocabulary.

In this paper we propose a simple method to obtain domain-specific sequences from unrestricted texts using statistical information only. This method is language-independent.

We had experiments on sequence extraction on email texts in Japanese, and succeeded in extracting significant semantic sequences in the test corpus. Wo tried morphological parsing on the test corpus with ChaSen, a Japanese dictionary-based morphological parser, and cxamined our system's efficiency in extraction of semantic sequences which were not recognized with ChaSen. Our system detected $69.06 \%$ of the unknown words correctly.
\end{abstract}

\section{Introduction}

Recognition of contained words is an important preprocessing for syntactic parsing. Word recognition is mostly done based on dictionary lookup, and unknown words often cause parse errors. Thus most of the researches have been done on fixed corpora with special dictionaries for the domain.

Part-of-spech(POS) tags are often used for term recognition. This kind of preprocessing is often time-consuming and causes ambiguity. When it comes to the corpus with high rate of unknown words it is not casy to do a fair parsing with dictionaries and rules.

Obtaining the contained terms and phrases correctly can be an efficient preprocessing. In this paper we propose a method to recognize domain-specific sequences with simple and noncosty processing, which enables the use of unrestiricted corpora for NLP tools.

We concentrate on building a tool for extracting meaningful sequences automatically with less preparation. Our system only needs a fair size of non-tagged training corpus of the target language. No restriction is required for the training corpus. Wo do not need any preprocessing for the training corpus.

Wo had experiments on cmail messages in Japanese and our system could recognize $69.06 \%$ of the undefined sequences of the test corpus.

\section{Japanese Characters and Terms}

Taking a word as a basic semantic unit simplifies the confusing tasks of processing real langllagos. However single words are often not a good unit regarding the meaning of the context, because of the polysemy of the words(Fung, 1998). Instead a phrase or a term can be taken as smallest. semantic units.

Most of the phrase/term extraction systems are based on recognizing noun phrases, or domain-specific terms, from large corpora. Argamon et al.(1998) proposed a memory-based approach for noun phrase, which was to learn paticrns with several sub-patierns. Ananiadou(1994) proposed a methodology based on torm recognition using morphological rules.

\subsection{Term Extraction in Japanese}

Japanese has no separator between words. On noun phrase extraction many researches have been done in Japanese as well, both stochastic and grammatical ways. In stochastic approaches $n$-gram is one of the most fascinating model. Noun phrase extraction(Nagao and Mori, 1994), word segmentation(Oda and Kita, 
1999) and diction extraction are the major issues. There also are many researches on segmentation according to the entropy. Since Japanese has a great number of characters use of the information of letters is also a very interesting issue.

\subsection{Characters in Japanese}

Unlike English, Japanese has great amount of characters for daily use. Japanese is special not only for its huge set of characters but its containing of three character types. Hiragana is a set of 71 phonctic characters, which are mostly used for function words, inflections and adverbs. Katakana is also a set of phonetic characters, each rolated to a hiragana character. The use is mainly restricted to the representation of foreign words. It's also used to represent pronunciations. Kanji is a set of Chinese-origin characters. There are thousands of kanji characters, and each kanji holds its own meaning. They are used to represent content words. We also use alphabetical characters and Arabic numerals.

\section{Overview}

This system takes Japanese sentences as input. It processes sentences one by one, and we obtain segments of the sentences which are recognized as meaningful sequences as output. The flow of this system is as follows(Figure 1): Our system

\begin{tabular}{ccc}
\hline TRAINING & EXTRACTION \\
\hline input (sentences) & input (a sentence) \\
$\Downarrow$ & $\Downarrow$ \\
cooccurrence & $\rightarrow$ & linking score \\
information & & calculation \\
& $\Downarrow$ \\
& sequence extraction \\
& $\Downarrow$ \\
& output (sequences) \\
\hline
\end{tabular}

Figure 1: The Flow of the System

takes one sentence as an input at one time, and calculates the scores between two neighboring letters according to the statistical data driven from the training corpus. After scoring the system decides which sequences to extract.

\subsection{Automatic Sequence Extraction}

Nobesawa et al. $(1996 ; 1999)$ proposed a sysiem which estimates the likelihood of a string of letters be a meaningful block in a sentence. This method does not need any knowledge of loxicon, and they showed that it was possible to segment; sentences in meaningful way only with statistical information between letiters. The experiment. was in Japancsc, and they also showed that the cooccurrence information between Japanese letters had enough information for estimating the connection of letters.

We use this point in this paper and had experiments on extracting meaningful sequences in email message texts to make up the lack of vocabulary of dictionaries.

\subsection{Scoring}

Our system introduces the linking score, which indicates the likelihood that two letticrs arc neighboring as a (part of) meaningful string(Nobesawa et al., 1996).

Only with neighboring bigrams it is impossible to distinguish the events ' $X Y$ ' in ' $A X Y B$ ' from ' $C X Y D$ '. Thus we introduce d-bigram which is a bigram cooccurrence information concerning the distance(Tsutsumi et al., 1993).

Expression (1) calculates the score between two neighboring letters;

$$
U K(i)=\sum_{d=1}^{d_{\text {max }}} \sum_{j=i \sim(d-1)}^{i} M_{d}\left(w_{j}, w_{j+d} ; d\right) \times g(d)
$$

where $w_{i}$ as an event, $d$ as the distance between two events, $d_{\max }$ as the maximum distance used in the processing (we set $d_{m a x}=5$ ), and $g(d)$ as the weight function on distance (for this system $g(d)=d^{-2}$ (Sano et al., 1996), to decrease the influence of the d-bigrams when the distance get longer (Church and Hanks, 1989)). When calculating the linking score between the letters $w_{i}$ and $w_{i+1}$, the d-bigram information of the letter pairs around the target two (such as $\left(w_{i-1}\right.$, $\left.w_{i+2} ; 3\right)$ ) are added.

Expression (2) calculates the mutual information between iwo events with d-bigram data;

$$
M M_{d}(x, y ; d)=\log _{2} \frac{P(x, y ; d)}{P(x) P(y)}
$$

where $x, y$ as events, $d$ for the distance between two events, and $P(x)$ as the probability.

\subsection{Sequence Extraction}

Using the linking score calculated according to the statistical information, our system searches for the sequences to extract (thus we call our system LSE(linky sequence extraction) system). 
Figure 2 shows an cxample graph of the linking scores for a sentence. Each alphabet leticr on the $\mathrm{x}$-axis stands for a letiter in a sentence.

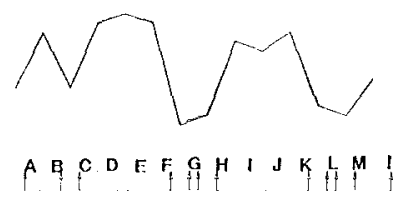

Figure 2: The Score Graph

The linking scores between two neighboring letters are dotted on the graph on the $y$-axis. Since the linking score gets higher when the pair has stronger connection, the mountain-shaped lines may get considered as unsegmentable blocks of letters. The linking scores of the pairs in longer words/phrases can be higher with the influence of the statistical information of other letter pairs around them. On the other hand, the linking score between two letters which are accidentally neighboring gets lower, and it makes valley-shaped point on the score graph. Our system extracts the mountainshaped parts of the sentence as the "linky sequences', which is considered to be meaningful according to the statistical information. In $\mathrm{ex}-$ ample Figure 2, strings $A B, C D E F$ and $H I J K$ might be extracted.

The height of mountains are not fixed, according to the likelihood of the letters blocked as a string. 'Thus we need a threshold to decide strings to extract according to the required size and the strength of connection. With higher threshold the strings gets shorter, since the higher linking score means that the ncighboring letters can be connected only when they have stronger connection between them.

\subsection{How the System Uses the Statistical Information}

Figure 3 shows the example graph on a sentence “お元気ですかか? [o-gen-ki-de-su-ka-?]” (: How are you?)(Sano, 1997). Each graph line indicaties the linking score of the sentence after learning some thousands of sentences of the target domain (for this graph we used a postcard corpus as the target domain, and for the base domain we took a newspaper corpus). When the system have no information on the postcard domain, the system could indicate that only the pair of letiers "元父i (gen-ki)" is connectable (there is a mountain-shaped line for this pair). Obtaining the information of posticard corpus, the linking scores of every pair in this sentence get bigger, to make higher mountain. And the shape of the mountain also changes to a flat one mountain which contains whole sentence from a steep mountain with deep valleys.

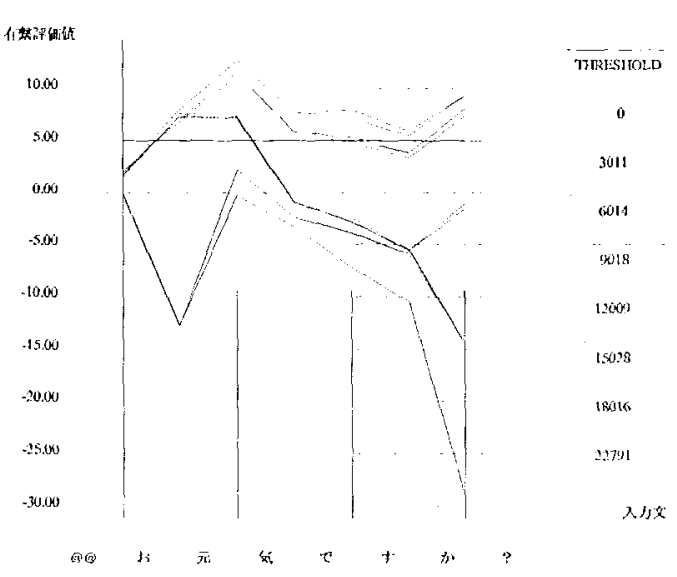

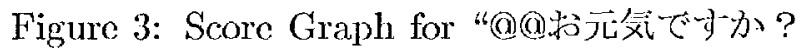
(@@-o-gen-ki-de-su-ka-?: How are you?)"

\section{Experiments}

Wo had experiments on extracting semantic scquences based only on letter cooccurrence information.

We tried a dictionary-based Japanese morphological parser ChaSen ver. 1.51(1997) on the test corpus as well to check sequences which a dictionary-based parser can not recognize.

\subsection{Corpus}

We chose email messages as the corpora for experiments of our system. Email messages are mostly written in colloquialism, especially when they are written by younger people to send to their friends. In Japancse colloquialism has casual wording which differs from literary style. Casual wording contains emphasizing and terms not in dictionary such as slangs. In English an emphasized word may be writien in capital lettcrs, such as in "it SURE is not true", which is easily connected to the basic word "sure". We do the same kind of letter type changes in Japanese for emplasizing, however, since the relationship between letter types is not the same as English, it is not easy to connect the emphasized terms and the basic terms. 


\subsubsection{Training Corpus}

The training corpus we used to extract statistical information is a set of email messages sent: between young female friends during 1998 to 1999. This corpus does not cover the one used as the test corpus. All the messages were sent to one receiver, and the number of senders is 17. The email corpus contains 351 email messages, which has 7,865 sentences $(176,380$ letters, i.e. 22.4 letters per sentence on average).

We did not include quotations of other emails in the training corpus to avoid double-counting of same sentences, though email messages often contain quotations.

\subsubsection{Test Corpus}

The test corpus is a set of cmail messages sent between young female friends during 1999. This corpus is not a part of the training corpus. All the messages were sent to one receiver, and the number of senders is 3 . This corpus contains 1,118 sentences $(24,160$ letters, i.e. 21.6 letters per sentence on average).

\subsection{Preliminary Results}

Figure 4 shows the distribution of the linking scores. The average of the scores is $\mathbf{0 . 3 4}$. The pairs of letters with higher linking scores are treated as highly 'linkable' pairs, that is, pairs with strong connection according to the statistical information of the domain (actually of the training corpus).

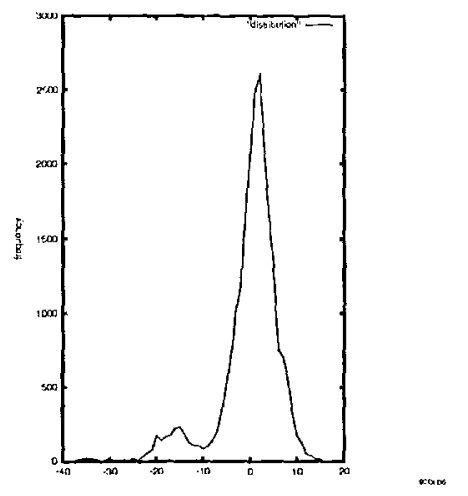

Figure 4: Score Distribution

Pairs of letters with high scores are mainly found in high-scored sequences (Table 1).

Table 1 shows a part of the scquences extracted with our system using letter cooccurrence information. The threshold of extraction for Table 1 is 5.0 .
Table 1: Sequences Extracted Based on Letter Cooccurrence

\begin{tabular}{|c|c|c|}
\hline sequence & meaning & $\begin{array}{c}\text { frequency } \\
\text { (with scores over } 5.0 \text { ) }\end{array}$ \\
\hline$\ldots a$ & …... & 72 \\
\hline そう & so & 52 \\
\hline けど $a$ & but & 48 \\
\hline 3. $5^{a}$ & therefore & 43 \\
\hline メール & mail & 39 \\
\hline (笑) & (laugh) & 36 \\
\hline ワタジ & I & 29 \\
\hline それ & it & 26 \\
\hline$\ldots a a$ & ...... & 25 \\
\hline 自分 & myself & 20 \\
\hline ホット $a$ & net/Internet & 20 \\
\hline$! ! a$ & $! !$ & 16 \\
\hline リンク & link & 15 \\
\hline 文暀 & friend & 13 \\
\hline
\end{tabular}

These sequences which extracted frequently are the ones often use in the target domain.

\subsection{Undefined Words with ChaSen}

Since ChaSen is a diciionary-based system, it outputs unknown strings of letters as they are, with a tag 'undefined word'.

Table 2 shows the number of sequences which ChaSen resulted as "undefined words". The row 'undefined words' indicates the sequences which were labeled as 'undefined word' with Chasen, and the row 'parsing errors' stands for the sequences which were not undefined words with ChaSen but not segmented correctily ${ }^{1}$. The extraction threshold is 0.5 .

ChaSen had 627 undefined words as its output. Since the test corpus contains 1,118 sentences, $56.08 \%$ of the sentences had an undefined word on average. As it is impossible to divide an undefined sequence into two undefined words, when two or more undefined sequences are neighboring they are often connected into one undefined word ${ }^{2}$ Thus the real number of undefined sequences can be more than counted. Table 2 shows that our system on statistical information can be a help to recover $69.06 \%$ of the undefined sequences detected by ChaSen.

\footnotetext{
${ }^{1}$ Since our system is not to put POS tags, we do not count tagging errors with ChaSen (i.c., we do not contain tagging errors in the 'parsing errors').

${ }^{2}$ ChaSen can divide two neighboring undifined scquences when the letter types of the sequences differs.
} 
Table 2: Undefined Words with ChaSen

\begin{tabular}{crrrr}
\multicolumn{2}{c}{ undefined words } & \multicolumn{3}{c}{ w/ LSE system } \\
frequency & \#total & suc..$^{a}$ & part. ${ }^{b}$ & failed \\
\hline over 10 & 281 & 230 & 7 & 41 \\
$3-9$ & 143 & 100 & 13 & 30 \\
2 & 56 & 43 & 4 & 9 \\
1 & 147 & 60 & 44 & 43 \\
\hline total & 627 & 133 & 68 & 126 \\
& & $69.06 \%$ & $10.85 \%$ & $20.10 \%$ \\
\hline
\end{tabular}

a suc: succeeded to extract

${ }^{\iota}$ part.: partially extracted

Table 2 also shows that this system has better precision with the sequences with larger frequency. For the sequences with frequency over 10 times (in the test corpus), $81.85 \%$ of the sequences have extracted correctly. Ignoring sequences which appeared in the test corpus once, the rate of correct extraction rose up to $77.71 \%$.

Table 3 shows how our system worked with the sequences which are found as undefined words with ChaSen parsing system. The threshold for extraction is 0.5 . Table 3 shows that the

Table 3: Categories of undefined Words

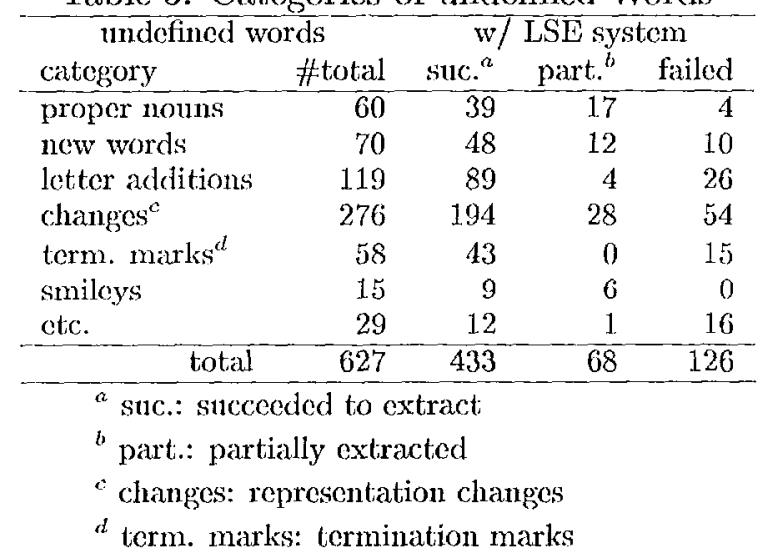

biggest reason for the undefined words are the problem of the representation. As described in Section 4.3.2, we change the way of description when we want to emphasize the sequence. The pronunciation extension with adding extra vowels or extension marks is also for the same reason. Adding these two categories, 356 sequences out of 627 undefined words $(56.78 \%)$ are caused in this emphasizing.

Termination marks as undefined words contain sequences such as "......" and "!!" ". The termination marks not in dictionary often indicate the impression, such as surprise, happiness, considering and so on.
New words including proper nouns are the actual 'undefined words'. ChaSen had 130 of them as its output, that is $20.73 \%$ of the undefined words.

\subsubsection{Letter Types in Undefined Words}

Table 4 shows the types of letters included in the 'undefined words' with ChaSen. The figures indicate the numbers of letters.

We had 627 undefined words in the test corpus with ChaSen (Table 2), which contain 1,493 letters totally. The average length of the undefined words is thus $2.38 .70 .40 \%$ of the letters in

Table 4: Letter Types of Undefined Words

\begin{tabular}{|c|c|c|c|c|c|}
\hline \multicolumn{3}{|c|}{ undefined words } & \multicolumn{3}{|c|}{ w/ LSE system } \\
\hline type & variety & \#total & suc. ${ }^{a}$ & part." & failed \\
\hline $\operatorname{kanjji}$ & 1. & 19 & 19 & $\overline{0}$ & 0 \\
\hline hiragana & 12 & 200 & 155 & 7 & 38 \\
\hline katakana & 73 & 1051 & 712 & 188 & 151 \\
\hline numeral & 1 & 1 & 0 & 1 & 0 \\
\hline alphabet & 23 & 122 & 43 & 72 & 7 \\
\hline symbol & 22 & 100 & 39 & 37 & 24 \\
\hline & total & 1493 & 968 & 305 & 220 \\
\hline
\end{tabular}

undefined words werc katakana letters(Table 4), which are phonetic and often used for describing new words. Katakana letters are also often used for emphasizing sequences.

On the other hand, there was only one letter cach for kanji and numeral figure. That is because cach kanji letter and numeral figure has its own meaning, and those letiters are mostly found in the dictionary, even though the tags are not semantically correct. Or, as for kanji letiters, it sometimes can be tagged with incorrecl segmentation ${ }^{3}$. Thus undefined words in kanji lelters are not counted as 'undefined words' mostly, and instead they cause segmentation failure(Section 4.4).

\subsubsection{Representation Changes}

Since Japanesc have two phonetic character sets, we have several ways to represent one term; in kanji (if there is any for the term), in hiragana, in katakana, or several character type mixed. It is also possible to use Romanization to represent a term.

\footnotetext{
3 “この [ko-no](:this)/世外 [sc-kai] (:the world)" is incorrectly segmented as "LOt世 [ko-no-yo](:the present life)/ 罚 [kai](:world)"; "kono yo" is a fixed phrase, and "kai" is a suffix for a noun to put the meaning of "the world of", c.g. "文学浆 (:the literary world)"
} 
Table 5 shows the numbers of ChaSen crrors according to the representation change. Most of

Table 5: Undefined Words because of Representation Changes

\begin{tabular}{|c|c|c|c|c|}
\hline \multicolumn{2}{|c|}{ undefined words } & \multicolumn{3}{|c|}{ w/ LSE system } \\
\hline subcategory & \#total & suc. $^{a}$ & part. $^{b}$ & failed \\
\hline term clianges & 40 & 33 & 3 & 4 \\
\hline katakana & 137 & 102 & 12 & 23 \\
\hline change \& katakana & 55 & 34 & 10 & 11 \\
\hline etc. & 44 & 25 & 3 & 16 \\
\hline total & 276 & 194 & 28 & 54 \\
\hline
\end{tabular}

a suc.: succeded to extract

${ }^{b}$ part.: partially extracted

the dictionaries have only one basic representation for one term as its entry ${ }^{4}$. However, in casual writing we sometimes do not; use the basic representation, to emphasize the torm, or just to simplify the writing.

\subsubsection{Pronunciation Extension}

In Japanese language we have many kinds of function words to put at the end of sentences (or sometimes 'bunsetsu' blocks). The function words for sentence ends are to change the sound of the sentences, to represent friendliness, ordering, and other emotions. These function words are not basically used in written texts, but in colloquial sentences.

In Japanese language we put extra letters to represent the lengthening of a phone. Since almost all Japanese phones have vowcls, to lengthen a phone for emphasizing we put extra vowels or extension marks after the letter. Table

Table 6: Extra Letters output as Undefined Words

\begin{tabular}{|c|c|c|c|c|c|c|c|c|c|}
\hline \multirow[t]{2}{*}{ letter } & あ & W & 5 & z & $お 3$ & 0 & 甲 & $y$ & total \\
\hline & a & $\mathbf{i}$ & 11 & $\mathrm{e}$ & o & $t$ & $t$ & $\mathbf{n}$ & \\
\hline suc. ${ }^{a}$ & 39 & 2 & 5 & 32 & 7 & 3 & 1 & 0 & 89 \\
\hline part. $^{b}$ & 0 & 0 & 0 & 0 & 0 & 4 & 0 & 0 & 4 \\
\hline failed & 5 & 1 & 4 & 2 & 1 & 7 & 5 & 1 & 26 \\
\hline total & 44 & 3 & 9 & 34 & $\overline{8}$ & 14 & 6 & 1 & 119 \\
\hline
\end{tabular}

6 shows that $74.79 \%$ of the small letters which resulted as undefined words with ChaSen could be salvaged as parts of scmantic sequences with our system.

\footnotetext{
${ }^{4}$ Dictionaries may have phonetic representations for the entries, not as headings.
}

These small letters in this table are extra letters to change the pronunciation; i.e. they are mostly not included in the dictionary. However they are actually a part of the word, since they could not be separated from the previous scquences.

\subsection{Segmentation Failure with ChaSen}

Table 7 shows the result of the extraction of sequences which ChaSen made parsing errors. It indicates that our system could recognize $70.88 \%$ of the sequences which ChaSen made parsing errors.

Table 7: Segmentation Failure with ChaSen

\begin{tabular}{crrrr}
\hline $\begin{array}{c}\text { undefined words } \\
\text { catcgory }\end{array}$ & \multicolumn{3}{c}{ W/ LSE system } \\
\hline A & 12 & 41 & 1 & 0 \\
B & 60 & 35 & 10 & 15 \\
C & 92 & 81 & 5 & 6 \\
D & 11 & 2 & 5 & 4 \\
E & 8 & 4 & 3 & 1 \\
F & 176 & 106 & 37 & 33 \\
G & 19 & 10 & 5 & 4 \\
H & 257 & 154 & 73 & 30 \\
I & 253 & 233 & 6 & 14 \\
J & 115 & 82 & 19 & 14 \\
\hline total & 941 & 667 & 159 & 115 \\
& & $70.88 \%$ & $16.90 \%$ & $12.22 \%$ \\
\hline
\end{tabular}

A: sequences incl. alphabetical characters

B: sequences incl. numeral figures

C: proper nouns

D: new words excl. proper nouns

F: fixed locutions

F: sequences with representation changes

$\mathrm{G}$ : sequences in other charactor types

II: emphasized expressions

I: termination marks

J: parsing crrors

${ }^{a}$ suc.: succeeded to extract

" part.: partially cxtracted

Calegory $\mathrm{F}$ is for the sequences which changed their representations according to the tcrms' pronunciation changes for casual use. For $c x-$ ample, "やっł゚ [ya-p-pa]" is a casual form of "や はり [ya-ha-ri](: as I thought)". In casual talking using original term "yahari" sounds a little too polite. Some common casual forms are in dictionaries, but not all.

For the category $B$, our system failed to cxtract 25 sequences. All the sequences in B are with counting suffixes. 12 sequences out of the 
25 could not be connected with the counting suffixes, e.g. " 3 O 1 [ [3-0-nichi](: 30 days, or, the 30th day)" got over-segmented between zero and the suffix. We have a big variety of counting suffixes in Japanese and since our system is only on letter cooccurrence information we could not avoid the over-segmentation.

Category $\mathrm{G}$ indicates the sequences which are writicen in other character types for emphasizing. The major changes are: (1) to write in hiragana characters instead of kanji characters, and (2) to write in katakana characters to emphasize the ticrm.

\section{Conclusion}

Dictionary-based NLP tools often have worse precision with texis written in casual wordings and texts which contain many domain-specific terms. 'Ierm recognition system available for any corpora as a preprocessing enables the use of NLP tools on many kinds of texts.

In this paper we proposed a simple method for term recognition based on statistical information. We had experiments on extracting semantically meaningful sequences according to the statistical information drawn from the training corpus, and our: system recognized $69.06 \%$ of the sequences which were tagged as undefined words with a conventional morphological parser.

Our system was efficiont in recognizing different representalions of terms, proper noums, and other casual wording phrases. This helps to salvage semantically moaningful sequences not in dictionaries and this can be an efficient preprocessing.

\section{Future Work}

In this paper we proposed a simple term recognition method based only on statistical information. There may be several ways to combine the extracted sequences with the dictionaries. We may need to put POS tags to the sequences for the use with other NLP tools. We expect that we can use tagging tools for this.

This system we propsed is languageindependent. For example, we can use this system on English to extract English sequences which appeared frequently in the training corpus, such as proper nouns.

\section{References}

Sophia Ananiadou. 1994. A Methodology for Automatic: Torm Rocognition. Coling-94, pages 10341038.

Shlomo Argamon, Ido Dagan, and Yuval Krymolowski. 1998. A Memory-Based Approach to Learning Shallow Natural Language Patterns. Coling-ACL'98, pages 67-73, August.

Kenneth W. Church and Patrick Hanks. 1989. Word $\Lambda$ ssociation Norms, Mutual Information, and Lexicography. The 27th Annual Conference of the Association of Computational Linguistics.

Pascalc Fung. 1998. Extracting Koy Terms from Chinese and Japanese texts. The International Journal on Computer Processing of Oriental Language, Special Issue on Information Retrieval on Oriental Languages.

Yuji Matsumoto, Akira Kitauchi, Tatsuo Yamashita, Yoshitaka Hirano, Osamu Imaichi, and Tomoaki Imamura. 1997. Japaneso? Morpholofical Analysis System ChaSen 1.51 Manual. Technical report, Nara Institute of Science and Technology. ht. hy://actus.aistnara.ac.jp/lab/nlt/chasen.html.

Makoto Nagao and Shinsulo Mori. 1994. $\Lambda$ New Method of N-gram Statisties for Large Number of $\mathrm{n}$ and Automatic Extraction of Words and Phrases from Large Text Data of Japanese. Coling-9/, pages 611-615, August.

Shiho Nobesawa, Junya T'sutsumi, Da Jiang Sun, Tomohisa Sano, Kongo Sato, and Masalazan Nalanishi. 1996. Segmenting; Sentoness into Linky Strings Using D-bigram Statistics. Coling-96, pages 586 591, August.

Shiho Nobesawa, Hiroaki Saito, and Masalazz1 Nakanishi. 1999. String Extraction Based Only on Statistic Linkability. ICCPOL'D9, pages 23 28, March.

Hiroki Oda and Konji Kita. 1999. A CharacterBased Japanese Word Segmonter Using a PPM*Based Language Model. ICCPOI,'99, pages 527532, March.

Tomohisa Sano, Junya Tsutsumi, Da Jiang Sun, Shiho Nobesawa, Kengo Sato, Kumiko Omori, and Masakazu Nakanishi. 1996. An Experimont on Good Usages of D-bigram Statistics in Natural Language Evaluation. 2nd Annual Mecting of the ANLP (NLPO6), pages 185-188. Written in Japanese.

Tomohisa Sano. 1997. Natural Language Processing Using Dynamic Statistical Information. Mastcr's thesis, Keio University. Written in Japanese.

Junya Tsutsumi, Tomoaki Nitta, Kotaro Ono, and Shiho Nobesawa. 1993. A Multi-Lingual Translation System Based on $\Lambda$ Statistical Model. JSAI Technical report, SIG-PPAI-9302-D, pages 7-12. Written in Japanese. 